\title{
Motion Analysis and Simulation of Crank Rocker Mechanism
}

\author{
Sun Jian \\ Jiangsu Key Laboratory of Large Engineering Equipment Detection and Control, School of Mechanical \& \\ Electrical Engineering \\ Xuzhou Institute of Technology \\ Xuzhou, China \\ e-mail: sunjian751@163.com
}

\begin{abstract}
Crank rocker mechanism is a common mechanism, widely used in sewing machine, mixer, shaper and other equipment. Structure analysis and Mathematical modeling were carried out on the working device of mechanism, then, based on software ADAMS, the simulation model of the crank rocker mechanism is established to carry out the kinematics simulation analysis. Through analysis, we can get the kinematics characteristics of each rod of crank rocker mechanism, and can well predict the performance of mechanism. The research method of this paper can provide a reference for the research of movement precision and mechanical design for the mechanism.
\end{abstract}

Keywords-motion analysis; simulation; crank rocker mechanism; ADAMS; kinematics

\section{INTRODUCTION}

At the institutional science, there are three kinds of Linkage application that converts the input and output motion, rigid guidance of connecting rod and trajectory of a point on the rod[1]. Because of the diversity of component motion form and the connecting rod curve of linkage, which can be widely used for engineering, so the design problems of linkage mechanism is still widely attention and deeply research scholars at home and abroad. Crank rocker mechanism is a common mechanism, widely used in sewing machine, mixer, adjustment mechanism on the radar, shaper and other equipment, which can make the whole week rotary motion into reciprocating swing, also can make reciprocating swing into rotary motion[2]. In this paper, based on the dynamic analysis of the crank rocker mechanism, the simulation model of the mechanism is established based on the multi-mechanical system simulation software ADAMS. Through simulation, the motion curve of the follower motion is obtained to understand the follower motion and predict the performance of mechanism. Then, the transmission angle of the mechanism has been optimized.

\section{KINEMATICS ANALYSIS}

For planar four-bar mechanism, when the sum of shortest and longest rod is less than or equal to the sum of other two rods, the mechanism exists crank. At this time, when the neighbors rod of the shortest rod is crank, the institution known as crank rocker mechanism.

As shown in Fig .1, The crank rocker mechanism mainly composed by crank, connecting rod, rocker and frame. Where $\mathrm{O}, \mathrm{A}, \mathrm{B}$ and $\mathrm{C}$ are each link point. $L_{1}, L_{2}$ and
$L_{3}$ represent the length of crank, connecting rod and rocker, respectively. $G_{1}, G_{2}$ and $G_{3}$ represent mass center position of each component. $\theta_{1}, \theta_{2}$ and $\theta_{3}$ are the angles between crank, connecting rod, rocker and forward $\mathrm{x}$ axis. $L_{4}$ is the length of the frame[3].

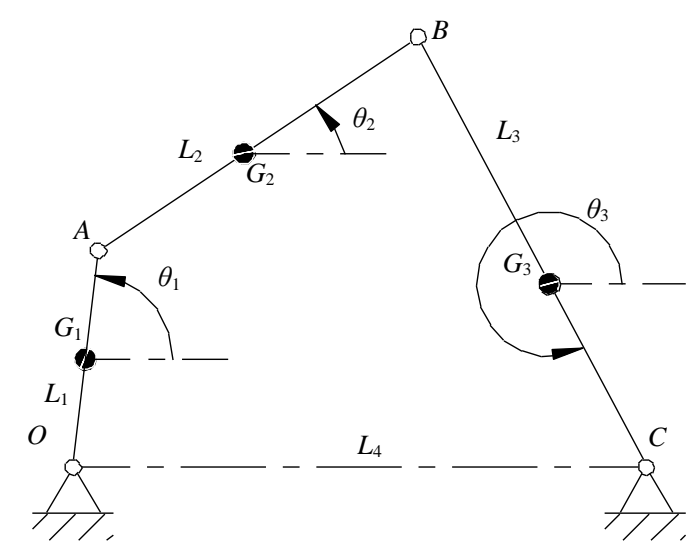

Figure 1. Schematic diagram of crank rocker mechanism

Kinematical equation of the mechanism is as

$$
\left\{\begin{array}{l}
L_{1} \sin \theta_{1}+L_{2} \sin \theta_{2}-L_{3} \sin \theta_{3}=0 \\
L_{1} \cos \theta_{1}+L_{2} \cos \theta_{2}-L_{3} \cos \theta_{3}-L_{4}=0
\end{array}\right.
$$

The centroid of each component can be written as

$$
\begin{gathered}
{\left[\begin{array}{l}
x_{G_{1}} \\
y_{G_{1}}
\end{array}\right]=\frac{L_{1}}{2}\left[\begin{array}{l}
\cos \theta_{1} \\
\sin \theta_{1}
\end{array}\right]} \\
{\left[\begin{array}{l}
x_{G_{2}} \\
y_{G_{2}}
\end{array}\right]=L_{1}\left[\begin{array}{l}
\cos \theta_{1} \\
\sin \theta_{1}
\end{array}\right]+\frac{L_{2}}{2}\left[\begin{array}{l}
\cos \theta_{2} \\
\sin \theta_{2}
\end{array}\right]} \\
{\left[\begin{array}{l}
x_{G_{3}} \\
y_{G_{3}}
\end{array}\right]=L_{1}\left[\begin{array}{l}
\cos \theta_{1} \\
\sin \theta_{1}
\end{array}\right]+L_{2}\left[\begin{array}{l}
\cos \theta_{2} \\
\sin \theta_{2}
\end{array}\right]+\frac{L_{3}}{2}\left[\begin{array}{l}
\cos \theta_{3} \\
\sin \theta_{3}
\end{array}\right]}
\end{gathered}
$$

The angle of the rocker is given, 


$$
\theta_{3}=\cos ^{-1}\left(\frac{L_{1} \sin \theta_{1}+L_{2} \sin \theta_{2}}{L_{3}}\right)
$$

Time-derivatives of mass center positions for moving links are given, respectively,

$$
\begin{gathered}
{\left[\begin{array}{c}
\dot{x}_{G_{i}} \\
\dot{y}_{G_{i}}
\end{array}\right]=\omega_{1}\left[\begin{array}{l}
\frac{\partial x_{G_{i}}}{\partial \theta_{1}} \\
\frac{\partial y_{G_{i}}}{\partial \theta_{1}}
\end{array}\right]} \\
{\left[\begin{array}{c}
\ddot{x}_{G_{i}} \\
\ddot{y}_{G_{i}}
\end{array}\right]=\alpha_{1}\left[\begin{array}{l}
\frac{\partial x_{G_{i}}}{\partial \theta_{1}} \\
\frac{\partial y_{G_{i}}}{\partial \theta_{1}}
\end{array}\right]+\omega_{1}^{2}\left[\begin{array}{l}
\frac{\partial^{2} x_{G_{i}}}{\partial \theta_{1}^{2}} \\
\frac{\partial^{2} y_{G_{i}}}{\partial \theta_{1}^{2}}
\end{array}\right]}
\end{gathered}
$$

The angle velocity and acceleration of the rocker are given in the following form,

$$
\begin{gathered}
\dot{\theta}_{3}=\omega_{1} \frac{\partial \theta_{3}}{\partial \theta_{1}} \\
\ddot{\theta}_{3}=\alpha_{1} \frac{\partial \theta_{3}}{\partial \theta_{1}}+\omega_{1}^{2} \frac{\partial^{2} \theta_{3}}{\partial \theta_{1}^{2}}
\end{gathered}
$$

Where $\omega_{1}$ is the angle velocity of the crank, $a_{1}$ is the angle acceleration of the crank.

According to the above formulas, substituting the known parameters, then the kinematic characteristics for each member of the institutions can be obtained[4].

\section{SiMULATION MODEL}

According to the motion characteristics, crank rocker mechanism can be divided into two types: I and II type. According to the research of literature [5], when the length of each component of the crank rocker mechanism meet the formula: $L_{1}^{2}+L_{4}^{2}<L_{2}^{2}+L_{3}^{2}$, this mechanism is called I type crank rocker mechanism; when the length meet the formula: $L_{1}^{2}+L_{4}^{2}>L_{2}^{2}+L_{3}^{2}$, this mechanism is called II type crank rocker mechanism. This study, We conducted the research to the I type and II type mechanism respectively[6]. The parameters of the I type and II type mechanism are shown in table 1 . The azimuth $\theta_{1}$ of driving member 1 is $0^{\circ} \sim 360^{\circ}$, and rotation with constant angular velocity $\omega_{1}=1 \mathrm{rad} / \mathrm{s}$.

Based on the above dynamic analysis, simulation model of the shaping machine is established by adopting the software ADAMS. The software-ADAMS could automatically established Lagrange equation based on the dynamic theory of multi-body system, and carry out the solution of kinematics and dynamics problems[7].
TABLE I. PARAMETERS OF MECHANISM

\begin{tabular}{|c|c|c|c|c|}
\hline Type & Parameters & Value & Parameters & Value \\
\hline \multirow{2}{*}{ I } & $L_{1} / \mathrm{mm}$ & 100 & $L_{2} / \mathrm{mm}$ & 291.5 \\
\cline { 2 - 5 } & $L_{3} / \mathrm{mm}$ & 255 & $L_{4} / \mathrm{mm}$ & 300 \\
\hline \multirow{2}{*}{ II } & $L_{1} / \mathrm{mm}$ & 150 & $L_{2} / \mathrm{mm}$ & 316.2 \\
\cline { 2 - 5 } & $L_{3} / \mathrm{mm}$ & 291.5 & $L_{4} / \mathrm{mm}$ & 450 \\
\hline
\end{tabular}

According to the given parameters of crank rocker mechanism, we can create 4 design points: point $\mathrm{O}$, point $\mathrm{A}$, point $\mathrm{B}$ and point $\mathrm{C}$ by using the user interface module, corresponding to the $\mathrm{O}, \mathrm{A}, \mathrm{B}, \mathrm{C}$ four hinge points of the mechanism[8]. Each bar of the crank rocker mechanism is attached to the design point to create, such as crank $A B$ is attached to the design points of point $\mathrm{A}$ and point $\mathrm{B}$, the length of the crank is the distance between the two design points. Frame AE is part of the earth that ADAMS software automatically creates.

Constraint is to determine the relationship of attachment and the relative motion between parts, this is the important point to analysis of kinematics, it is directly affect the accuracy of the analysis and simulation results that the constraints applying is correct or not. Then Gravity constraints are applied to the entire mechanism, rotation constraints are added between two members of the crank and the earth, the crank and the connecting rod, the connecting rod and the rocker, the rocker and the earth. The drive is applied on the crank, and the angular velocity is set to $1 \mathrm{rad} / \mathrm{s}$. The virtual prototyping model of the I type and II type mechanism are shown in Fig .2.

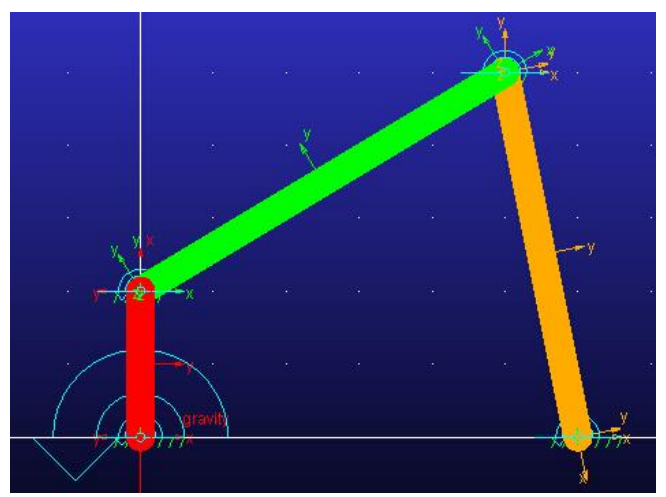

Figure 2. I type mechanism

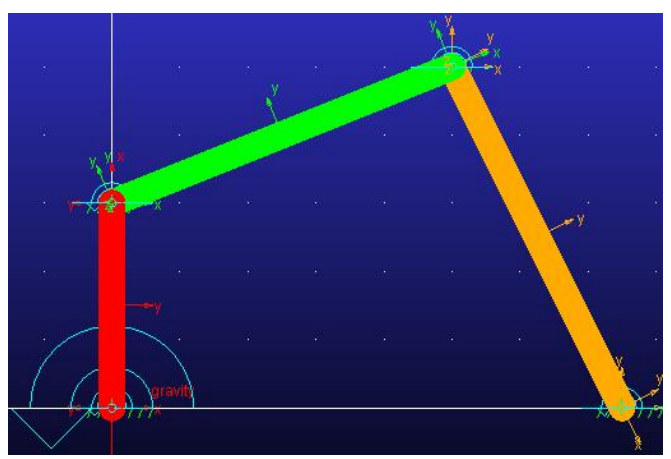

Figure 3. II type mechanism 


\section{SimUlation AND DisCUSSION}

\section{A. Simulation Analysis of I type Mechanism}

ADAMS provides some simulation tracing and debugging tools, in the process of debugging prototype simulation, it can use these tools to track the simulation results and timely troubleshooting[9]. Select the simulation tool icon in the main toolbox to display the interactive simulation analysis settings bar, in the "End-Time" field, enter 1, indicating that the simulation run time, In the "steps" field, enter 300, represents the number of steps throughout the simulation output. After completing the above Settings, press the run key start simulation analysis.

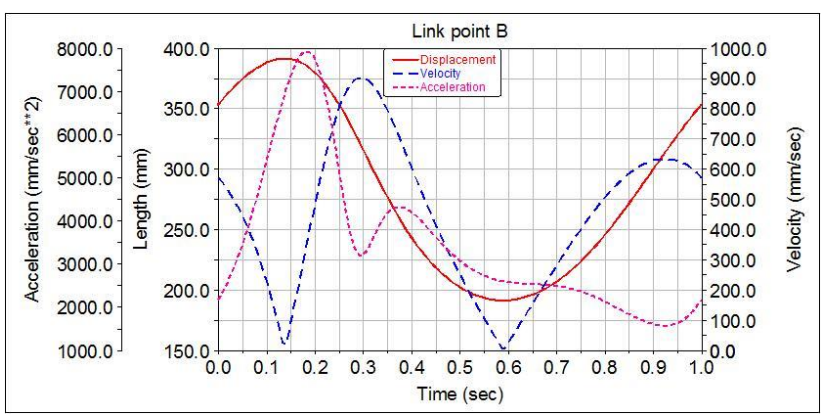

Figure 4. Kinematics curve of link point B

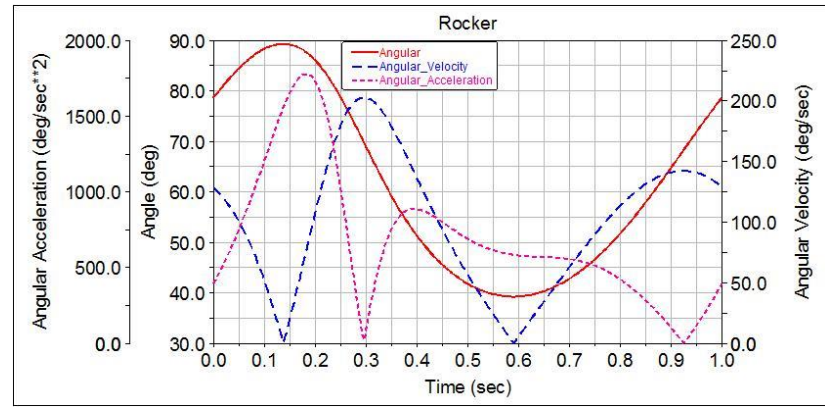

Figure 5. Kinematics curve of rocker

In the whole process of the simulation analysis[10], the computer can display real-time operation state of a prototype. Fig .4 shows the displacement, velocity and acceleration curves of link point B. Fig .5 represents the azimuth angle, angle velocity and angle acceleration curve of the rocker.

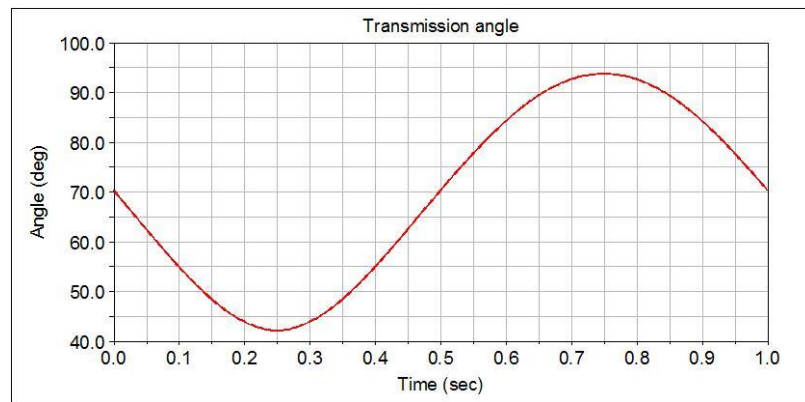

Figure 6. Transmission angle curve of I type mechanism
A measurement of angle is arranged between the connecting rod and rocker, then, we can get the transmission angle curve of crank rocker mechanism, as shown in Fig .6.

\section{B. Simulation Analysis of II type Mechanism}

According to the same method as the I type mechanism to simulate the II type mechanism. Fig .7 shows the displacement, velocity and acceleration curves of link point B. Fig .8 represents the azimuth angle, angle velocity and angle acceleration curve of the rocker. Fig .9 shows the transmission angle curve of II type mechanism.

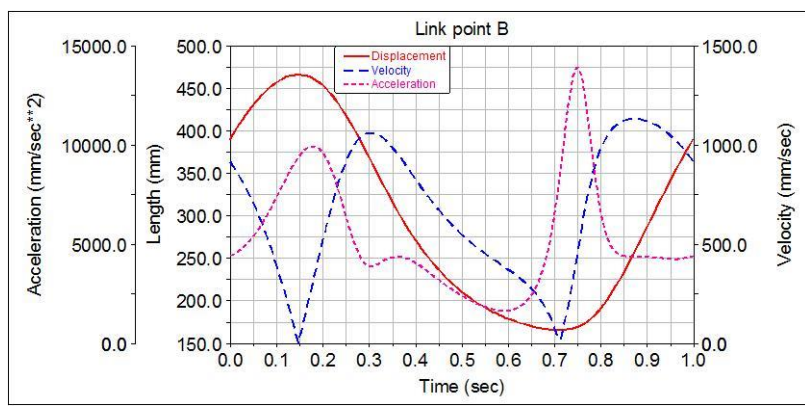

Figure 7. Kinematics curve of link point B

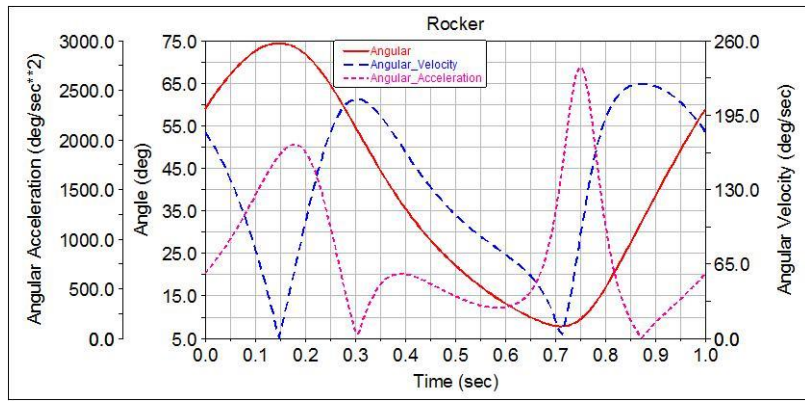

Figure 8. Kinematics curve of rocker

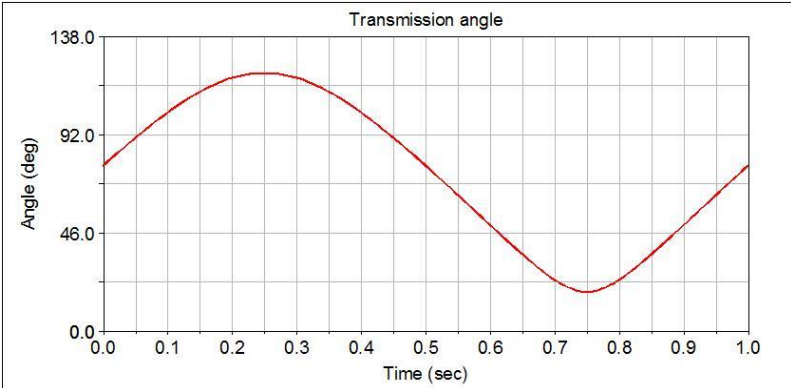

Figure 9. Transmission angle curve of II type mechanism

\section{Results and Discussion}

As shown in Fig .4 and Fig .7, the displacement curve of link point B of II type is similar to I type mechanism. However, for the velocity curve, we can find that the velocity of link point $B$ is faster in the anticlockwise rotation, and slower during the return stroke in Fig .4, but 
from Fig .7, the velocity of link point B is slower in the anticlockwise rotation, and faster during the return stroke, which represent that the quick return characteristics of I type and II type mechanism is different. From the acceleration curves of link point B, acceleration is larger at the beginning and decreases during the return stroke in Fig .4, however, there are two peaks of acceleration and greater during the return stroke in Fig .7. The kinematics curve as shown in Fig .5 and Fig .8 reflect the same characteristic. In Fig .5, with the maximum value minus the minimum value of angular curve, we can get the rocker pendulum angle of I type crank rocker mechanism which is $50.03^{\circ}$. Using the same method, the rocker pendulum angle of II type crank rocker mechanism is $66.54^{\circ}$.

Transmission angle is an important parameter in the mechanical design and denotes the quality of motion transmission in a mechanism, and it is mainly used to obtain the better results for various linkage applications. As shown in Fig .6, the transmission angle of I type mechanism has a minimum in the beginning and a maximum in return, and the minimum transmission angle is $42.27^{\circ}$. From Fig .9, The change of II type transmission angle is opposite with I type, and the minimum transmission angle of II type is $18.39^{\circ}$. In addition, We can also easily use the simulation model to analyze the other dynamics and predict the performance of mechanism.

\section{CONCLUSIONS}

In this paper, according to the structure principle of the crank rocker mechanism, the mathematical model is established. Based on the movement characteristics, the crank rocker mechanism is divided into I type and II type, then, the simulation model of I type and II type mechanism are established respectively and the kinematic process is simulated by the dynamics analysis software-ADAMS. It can be seen clearly from the kinematics curve of rocker, the angle velocity of rocker of I type mechanism is faster in the anticlockwise rotation, and slower during the return stroke, and II type mechanism is opposite. This method can also be used for kinematic analysis of other mechanism, which is conducive to screening and optimization of mechanism design scheme early.

\section{REFERENCES}

[1] L. Zhigang, "Optimized analysis of crank and rocker mechanism based on MATLAB," FOOD \& MACHINERY, vol. 30, Mar. 2014, pp. 114-117.

[2] Y. Jinhu, "Research of the Transmission Angle Function of Offset Crank Rocker Mechanism," Journal of Mechanical, vol. 38, Aug. 2014, pp. 43-46

[3] Y. Sudi, and X. Jinghua, "Kinematics and dynamicsanalysis for slotting machine six bar linkage", Journal of Anhui polytechnic University, vol. 29, Dec. 2014, pp. 27-31.

[4] G.Weidong, Theory of Machines and mechanisms, China Science Press, 2010.

[5] Q. Ruining, and L. Qingyun, "Some Propositions Related to Extreme Position Angle of Crank rocker Mechanisms and Their Applications," CHINESE JOURNAL OF MECHANICAL ENGINEERING, vol. 41, Jul. 2005, pp. 80-83.

[6] S.Youliang, "Optimization design of crank rocker mechanism basede on maximum of minimum transmission angle," JOURNAL OF MACHINE DESIGN, vol. 31, Jul. 2014, pp. 29-33.

[7] G, Zenghao, ADAMS2007 virtual prototyping technology, Chemical Industry Press, China, 2010.

[8] X.Yuan, "The Simulation and Analysis of the Movement of Crank Rocker Mechanism Based on Pro/E," Journal of Chongqing Industry \& Trade Polytechnic,” vol. 35, Mar. 2014, pp. 15-17.

[9] L.jixia, From entry to the master of the virtual prototype of ADAMS 2012, machine-building industry press, 2013.

[10] T. Xuemei, "The Script Simulation of Shifting Process in Transmission System," International Conference on Mechanic Automation and Control Engineering, 2010. 